\title{
The effect of photocopier types on line quality features of handwriting in multi-generational photocopies
}

\author{
OM Prakash Jasuja \\ ORCID: 0000-0002-2221-1242
}

Department of Forensic Science and Chemistry, Chandigarh University

Gharua, Punjab, India

\section{SURYAKANT Mishra}

ORCID: 0000-0001-5220-9066

Research Scholar, Department of Forensic Science, Punjabi University

Patiala, India

\section{MOHINDER SINGH}

ORCID: 0000-0002-2649-1616

Formerly Government Examiner of Questioned Documents

Central Forensic Science Laboratory, Hyderabad, India

\begin{abstract}
The present research was aimed at finding out the relative effect of advancement in photocopier technology on the analysis of handwriting line quality features in multi-generational photocopies. Five subsequent multi-generational photocopies of 50 signature samples from different individuals which were produced by 75 black and white photocopiers were used for line quality assessments. Variations in line quality features in different photocopy generations, such as: smooth edges or ragged edges, broken or continuous stroke, fine tapering changes, and some identification details of features like pen lifts,
\end{abstract}


retouching, and hesitation were carefully observed in sample photocopies, which were recorded according to the brand and model of photocopier machines, as well as their size and speed. The results of the study revealed that raggedness in line was often observed in third generation (G3) and higher generations, in samples produced by some low speed copiers (copying speed $<30 \mathrm{cpm}$ ) or very high speed copiers (copying speed $\leq 80 \mathrm{cpm}$ ). However, their line quality was still found to be good enough for handwriting analysis up to the fifth generation (G5) in photocopy samples produced by some medium speed copiers (copying speed 30 to $50 \mathrm{cpm}$ ). It was, however, observed that, usually, continuity of line and smoothness of line were appreciably lost in G3 and higher generations in photocopies which were produced by portable desktop printers. Exceptionally, certain artifacts that are usually found in some higher generation copies were conspicuously absent in all photocopy generations (G1-G5) which were produced by some advanced third generation photocopiers. Hopefully, the information obtained from the study will be found useful to document examiners when examining certain cases involving multi-generations of photocopier reproductions.

Keywords: photocopy, questioned documents, forensic science, handwriting examination, line quality of handwriting

\section{Introduction}

Forensic document examiners commonly scrutinize different types of questioned document problems such as identification of writing, security records, currency notes, authentication of stamps, etc., to solve the legal issues by analysing the original as well as non-original documents. Photocopying has become a common mode of document reproduction and is routinely used by most people. Photocopies could be accepted as secondary evidence under the Indian Evidence Act, 1872, in the case of disputed handwriting. Line quality is an important attribute of handwriting, often used to characterise the genuine and forged handwriting. It is derived from a combination of factors, including writing skill, speed, rhythm, freedom of movement, shading, and pen position. ${ }^{1}$ Maintaining smoothness of the writing line without losing identifying attributes is important in case of reproduction of documents. ${ }^{2}$ Some experts have expressed the view that multi-generation photocopying degrades

1 O. Hilton, Scientific examination of questioned documents, Boca Raton, FL 1993, pp. 361-388.

2 K.M. Koppenhaver, Forensic document examination: Principles and practice, Totowa, NJ 2007, pp. 83-84. 
the quality of documents. ${ }^{3}$ The present research was conducted to find out some new information on the effect of advancement in photocopier technology on the analysis of handwriting line quality features in multigenerational photocopies.

\section{Methodology}

For the present study, signature samples of 50 individuals were collected on A4 size paper using ballpoint pens. The original signature samples went through up to five subsequent generations of photocopy using 75 different photocopiers (Table 1). Photocopies of signatures were examined and compared with original samples using a magnifying lens $(5 \times)$ and stereomicroscope to find out the variations in line quality according to the brand, model, speed, and size of photocopiers.

Table 1. List of photocopiers used for the collection of black and white photocopy samples

\begin{tabular}{|l|l|c|c|c|c|c|}
\hline $\begin{array}{c}\text { Make } \\
\text { and model }\end{array}$ & Size & $\begin{array}{c}\text { Speed } \\
\text { CPM }\end{array}$ & $\begin{array}{c}\text { Input } \\
\text { resolution in } \\
\text { dot per inch }\end{array}$ & $\begin{array}{c}\text { Output } \\
\text { resoloution in } \\
\text { dot per inch }\end{array}$ & Drum & $\begin{array}{c}\text { AX } \\
{[\%]}\end{array}$ \\
\hline Canon IR 2270 & MS & 22 & $1200 \times 6001$ & $2400 \times 6001$ & OPC & 1 \\
\hline Canon IR 2270 & MS & 22 & $1200 \times 6001$ & $2400 \times 6001$ & OPC & 1 \\
\hline Canon IR 2270 & MS & 22 & $1200 \times 6001$ & $2400 \times 6001$ & OPC & 1 \\
\hline Canon IR 2270 & MS & 22 & $1200 \times 6001$ & $2400 \times 6001$ & OPC & 1 \\
\hline Canon IR 2270 & MS & 22 & $1200 \times 6001$ & $2400 \times 6001$ & OPC & 1 \\
\hline Canon IR 2830 & MS & 28 & $1200 \times 6001$ & $2400 \times 6001$ & OPC & 1 \\
\hline Canon IR 2870 & MS & 28 & $1200 \times 6001$ & $2400 \times 6001$ & OPC & 1 \\
\hline Canon IR 2870 & MS & 28 & $1200 \times 6001$ & $2400 \times 6001$ & OPC & 1 \\
\hline Canon IR 3025 & MS & 25 & $1200 \times 6001$ & $2400 \times 6001$ & OPC & 1 \\
\hline Canon IR 3245 & MS & 45 & $1200 \times 6001$ & $1200 \times 12001$ & OPC & 1 \\
\hline
\end{tabular}

${ }^{3}$ O. Hilton, op. cit.; R. Morris, Forensic handwriting identification, fundamental concepts and principles, San Diego 2000; M. Allen, Foundations of forensic document analysis: Theory and practice, Chichester 2016. 


\begin{tabular}{|c|c|c|c|c|c|c|}
\hline Canon IR 3235N & MS & 35 & $1200 \times 6001$ & $1200 \times 12001$ & $\mathrm{OPC}$ & 1 \\
\hline Canon IR 3235N & MS & 35 & $1200 \times 6001$ & $1200 \times 12001$ & $\mathrm{OPC}$ & 1 \\
\hline Canon IR 3235i & MS & 45 & $1200 \times 6001$ & $1200 \times 12001$ & $\mathrm{OPC}$ & 1 \\
\hline Canon IR 3300 & MS & 33 & $1200 \times 6001$ & $2400 \times 12001$ & $\mathrm{OPC}$ & 1 \\
\hline Canon IR 3300 & MS & 33 & $1200 \times 6001$ & $2400 \times 12001$ & $\mathrm{OPC}$ & 1 \\
\hline Canon IR 3300 & MS & 33 & $1200 \times 6001$ & $2400 \times 12001$ & $\mathrm{OPC}$ & 1 \\
\hline Canon IR 3300 & MS & 33 & $1200 \times 6001$ & $2400 \times 12001$ & $\mathrm{OPC}$ & 1 \\
\hline Canon IR 3300 & $\mathrm{MS}$ & 33 & $1200 \times 6001$ & $2400 \times 12001$ & $\mathrm{OPC}$ & 1 \\
\hline Canon IR 3300 & MS & 33 & $1200 \times 6001$ & $2400 \times 12001$ & $\mathrm{OPC}$ & 1 \\
\hline Canon IR 3300 & MS & 33 & $1200 \times 6001$ & $2400 \times 12001$ & $\mathrm{OPC}$ & 1 \\
\hline Canon IR 3320i & MS & 33 & $1200 \times 6001$ & $2400 \times 12001$ & $\mathrm{OPC}$ & 1 \\
\hline Canon IR 3530 & MS & 35 & $1200 \times 6001$ & $2400 \times 6001$ & $\mathrm{OPC}$ & 1 \\
\hline Canon IR 6000 & LS & 60 & $1200 \times 6001$ & $2400 \times 6001$ & $\mathrm{OPC}$ & 1 \\
\hline Canon IR 6000 & LS & 60 & $1200 \times 6001$ & $2400 \times 6001$ & $\mathrm{OPC}$ & 1 \\
\hline Canon IR 7095 & LS & 95 & $1200 \times 6001$ & $2400 \times 6001$ & $\begin{array}{l}\mathrm{OPC} / \\
\mathrm{ASi}\end{array}$ & 1 \\
\hline Canon IR 7095 & LS & 95 & $1200 \times 6001$ & $2400 \times 6001$ & $\begin{array}{l}\mathrm{OPC} / \\
\mathrm{ASi}\end{array}$ & 1 \\
\hline Canon IR 7095 & LS & 95 & $1200 \times 6001$ & $2400 \times 6001$ & $\begin{array}{l}\mathrm{OPC} / \\
\mathrm{ASi}\end{array}$ & 1 \\
\hline Canon IR 7105 & LS & 105 & $1200 \times 6001$ & $2400 \times 6001$ & A Si & 1 \\
\hline Canon IR 7105 & LS & 105 & $1200 \times 6001$ & $2400 \times 6001$ & A Si & 1 \\
\hline Canon IR 7200 & LS & 72 & $1200 \times 6001$ & $2400 \times 6001$ & A Si & 1 \\
\hline Canon IR 7200 & LS & 72 & $1200 \times 6001$ & $2400 \times 6001$ & A Si & 1 \\
\hline Canon IR 7200 & LS & 72 & $1200 \times 6001$ & $2400 \times 6001$ & A Si & 1 \\
\hline Canon IR 7200 & LS & 72 & $1200 \times 6001$ & $2400 \times 6001$ & A Si & 1 \\
\hline Canon IR 7200 & LS & 72 & $1200 \times 6001$ & $2400 \times 6001$ & A Si & 1 \\
\hline
\end{tabular}




\begin{tabular}{|c|c|c|c|c|c|c|}
\hline Canon IR 8070 & LS & 80 & $1200 \times 6001$ & $2400 \times 6001$ & A Si & 1 \\
\hline Canon IR 8070 & LS & 80 & $1200 \times 6001$ & $2400 \times 6001$ & A Si & 1 \\
\hline Canon IR 8500 & LS & 85 & $1200 \times 6001$ & $2400 \times 6001$ & A Si & 1 \\
\hline Canon IR 8500 & LS & 85 & $1200 \times 6001$ & $2400 \times 6001$ & A Si & 1 \\
\hline Canon IR 8500 & LS & 85 & $1200 \times 6001$ & $2400 \times 6001$ & A Si & 1 \\
\hline Canon MF 3010 & PD & 18 & $600 \times 600$ & $600 \times 600$ & $\mathrm{OPC}$ & 1 \\
\hline Canon MF 4320 & PD & 22 & $600 \times 600$ & $600 \times 600$ & $\mathrm{OPC}$ & 1 \\
\hline Canon MF 4412 & PD & 23 & $600 \times 600$ & $600 \times 600$ & $\mathrm{OPC}$ & - \\
\hline Canon MF 241 & PD & 38 & $600 \times 600$ & $600 \times 600$ & $\mathrm{OPC}$ & - \\
\hline Canon MF 241 & PD & 38 & $600 \times 600$ & $600 \times 600$ & $\mathrm{OPC}$ & - \\
\hline Develop 213 & PD & 21 & $600 \times 600$ & $600 \times 600$ & $\mathrm{OPC}$ & 1 \\
\hline HP 1005 & PD & 15 & $300 \times 300$ & $300 \times 300$ & $\mathrm{OPC}$ & - \\
\hline HP 1005 & PD & 15 & $300 \times 300$ & $300 \times 300$ & $\mathrm{OPC}$ & - \\
\hline Konica MB 215 & MD & 21 & $600 \times 600$ & $600 \times 600$ & $\mathrm{OPC}$ & 0.1 \\
\hline Konica MB 215 & MD & 21 & $600 \times 600$ & $600 \times 600$ & OPC & 0.1 \\
\hline Konica MB 215 & MD & 21 & $600 \times 600$ & $600 \times 600$ & $\mathrm{OPC}$ & 0.1 \\
\hline Konica MB 423 & MS & 42 & $600 \times 600$ & $600 \times 600$ & OPC & 0.1 \\
\hline $\begin{array}{l}\text { Konica } \\
\text { MB C458 }\end{array}$ & MS & 45 & $600 \times 600$ & $1800 \times 600$ & OPC & 0.1 \\
\hline $\begin{array}{l}\text { Konica } \\
\text { MB C552 }\end{array}$ & MS & 55 & $600 \times 600$ & $1800 \times 600$ & $\mathrm{OPC}$ & 0.1 \\
\hline Ky EM 2040 & PD & 40 & $600 \times 600$ & $600 \times 600$ & OPC & 1 \\
\hline Ky EM 2040 & PD & 40 & $600 \times 600$ & $600 \times 600$ & OPC & 1 \\
\hline Ку ЕМ 2040 & PD & 40 & $600 \times 600$ & $600 \times 600$ & OPC & 1 \\
\hline Ky EM 2040 & PD & 40 & $600 \times 600$ & $600 \times 600$ & OPC & 1 \\
\hline Ky EM 2040 & PD & 40 & $600 \times 600$ & $600 \times 600$ & OPC & 1 \\
\hline Ky FS 1035 & PD & 35 & $600 \times 600$ & $600 \times 600$ & OPC & 1 \\
\hline
\end{tabular}




\begin{tabular}{|l|l|l|l|l|l|c|}
\hline KyTA 300i & MS & 30 & $600 \times 600$ & $600 \times 600$ & OPC & 1 \\
\hline Samsung 4521 & PD & 20 & $600 \times 600$ & $600 \times 600$ & OPC & - \\
\hline Sharp AR 5620 & MD & 20 & $600 \times 600$ & $600 \times 600$ & OPC & - \\
\hline Sharp ARM 205 & MD & 20 & $600 \times 600$ & $600 \times 600$ & OPC & - \\
\hline Xerox V L 7030 & LS & 30 & $600 \times 600$ & $600 \times 600$ & OPC & 1 \\
\hline Xerox 440 & MS & 40 & $600 \times 600$ & $600 \times 600$ & OPC & 1 \\
\hline Xerox 440 & MS & 40 & $600 \times 600$ & $600 \times 600$ & OPC & 1 \\
\hline Xerox 440 & MS & 40 & $600 \times 600$ & $600 \times 600$ & OPC & 1 \\
\hline Xerox 440 & MS & 40 & $600 \times 600$ & $600 \times 600$ & OPC & 1 \\
\hline Xerox WC 5755 & LS & 55 & $600 \times 600$ & $4800 \times 6001$ & OPC & 1 \\
\hline Xerox WC 5755 & LS & 55 & $600 \times 600$ & $4800 \times 6001$ & OPC & 1 \\
\hline Xerox WC 5755 & LS & 55 & $600 \times 600$ & $4800 \times 6001$ & OPC & 1 \\
\hline Xerox WC 5755 & LS & 55 & $600 \times 600$ & $4800 \times 6001$ & OPC & 1 \\
\hline Xerox WC 5755 & LS & 55 & $600 \times 600$ & $4800 \times 6001$ & OPC & 1 \\
\hline Xerox WC 5790 & LS & 90 & $600 \times 600$ & $4800 \times 6001$ & OPC & 1 \\
\hline $\begin{array}{l}\text { Canon } \\
\text { NP 3050@ }\end{array}$ & MD & - & $150 \times 150$ & $150 \times 150$ & OPC & 1 \\
\hline
\end{tabular}

Abbreviations

MS: medium size multifunction floor standing copier

LS: large size multifunction floor standing copier

PD: portable desktop MF printer

MD: medium size desktop MF printer

CPM: copy per minute

@: analogue photocopier

A Si: amorphous silicon drum

OPC: organic photoconductor drum

$\mathrm{X}^{1}$ : interpolated resolution

AX: additional magnification 


\section{Results}

To facilitate systematic study, the used photocopiers have been divided into 14 different groups according to their make and model/series:

1. Canon IR series,

2. Canon MF series,

3. Develop Ineo 213,

4. HP 1005,

5. Konica MB series,

6. Kyocera EM series,

7. Kyocera FS 1035 ,

8. Kyocera TA 300i,

9. Samsung 4521,

10. Sharp AR series,

11. Xerox DC 440,

12. Xerox V L 7030,

13. Xerox WC series,

14. Canon NP 3050.

Photocopy quality variations were observed in different generations based upon the same line quality features such as: smooth/ragged edges, broken/continuous stroke, etc. These findings are presented below, according to the make and model/series of the photocopiers and including their sizes and speed.

Canon Image Runner (IR) series. Line quality in G1 and G2 was found good enough for the analysis of handwriting in all the copiers from this series. However, raggedness in line was often seen in G3 and higher generations of photocopies produced by some low speed copiers (copying speed $<30 \mathrm{cpm}$ ) such as Canon IR 2270, or very high speed copiers (copying speed $\leq 80 \mathrm{cpm}$ ), including Canon IR 8500 and Canon IR 7105. Artifacts such as thinning in line strokes were also seen in photocopy samples produced by high speed copiers. However, in photocopy samples produced by some medium speed copiers (copying speed 30 to $50 \mathrm{cpm}$ ) such as Canon IR 3320i, the line quality was found good enough for analysis up to G5 (Figure 1). 

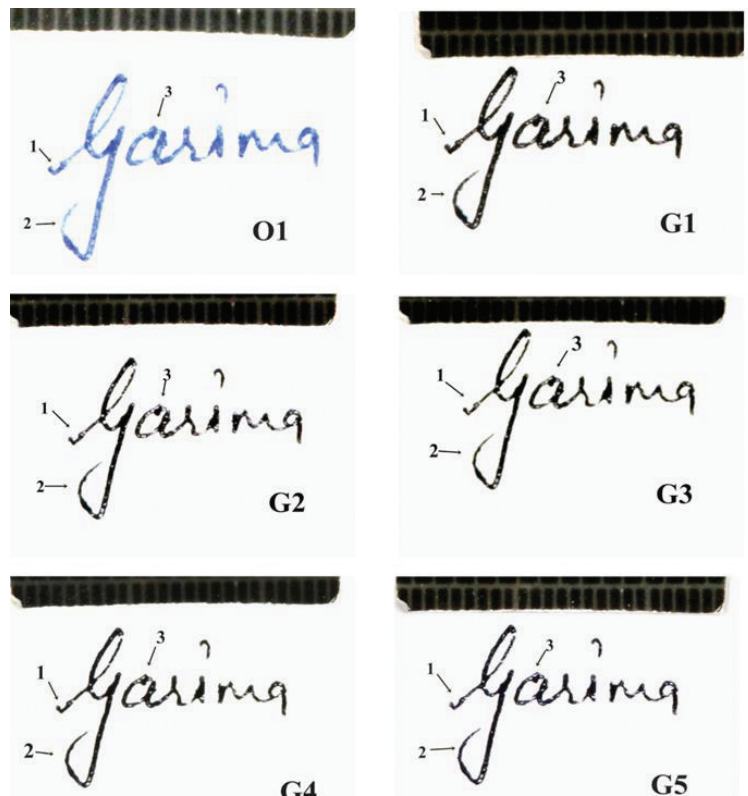

G4

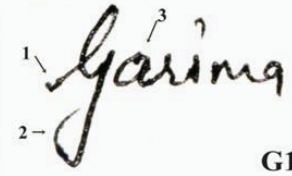

G1

G3

G5

Figure 1. Showing line quality in the original signature $(\mathrm{O} 1)$ and its photocopies $(\mathrm{G} 1$ to G5), line quality features such as (1) an inkless start with an ink blob at initial stroke, (2) a tapered end, and (3) an ink gooping could be identified in G1 to G5 (Canon IR 3235i)

Canon MF series printers. Portable desktop printers. Line continuity and smoothness were lost in G3 and further generations of photocopies. Overall line quality was not found good enough for forensic analysis of fine line quality features such as pen pause, retouching, etc. beyond G2.

Develop Ineo 213. A low speed desktop multifunction printer; line quality in photocopies was found similar to Canon MF series printers.

HP 1005 series. A portable desktop multifunction printer; line quality in photocopies was found similar to Canon MF series printers.

Konica MB series. This series includes one of the best photocopiers available on the market. Artifacts such as breaks and raggedness were not found in any photocopy generations G1 to G5; normally, line quality was deemed good enough for analysis in G1 to G5. Line quality features such 
as fine tapering strokes, ink gooping, hesitations, retouching, or excessive ink deposition could be identified up to G5.

Kyocera EM 2040. A medium speed portable desktop printer. Average photocopy quality was found in early generations G1 and G2, after that intense raggedness in line edges had appeared (Figure 2).
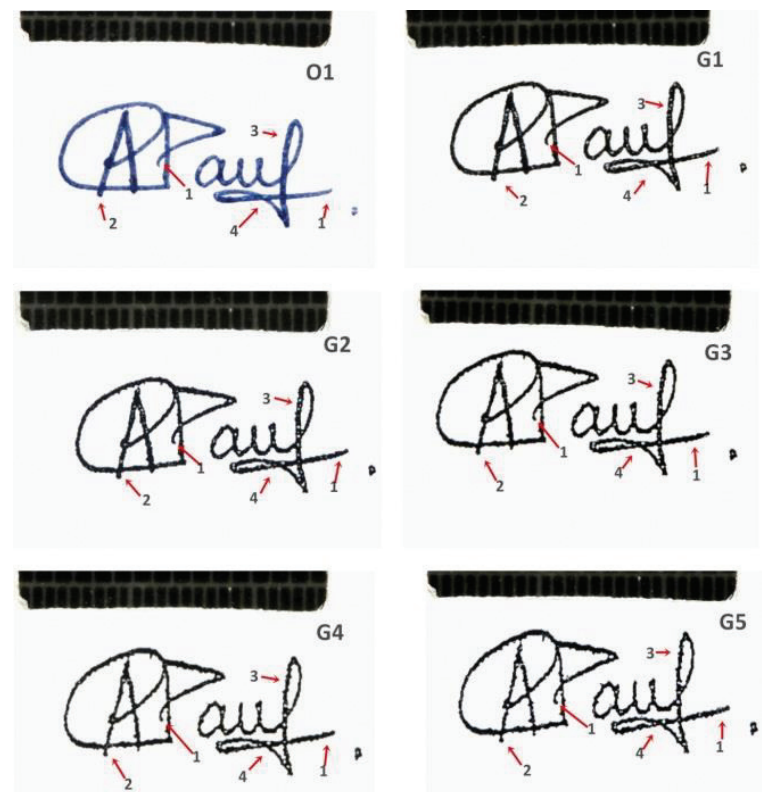

Figure 2. Showing deterioration in line smoothness in G1 to G5; line features such as (1) tapering, (2) a blunt initial, (3) a smooth stroke, and (4) the direction of pen movement are identifiable in G1 and G2; thereafter, intense raggedness is seen in G3 to G5 (Kyocera EM 2040)

Kyocera FS 1035. A medium speed desktop printer. Line quality in photocopies was almost similar to Ky EM 2040.

Kyocera TA 300i. A floor standing multifunction copier. Raggedness in strokes was found in subsequent generations of photocopies, as well as, at places, large dots forming in photocopied strokes. Thus, some important line quality features such as tapering, ink goopings, pen lifts, retouching, and joining were not identifiable. 
Samsung SCX 4521F. A low speed portable desktop printer. Faint or light inked strokes of original handwriting could not be reproduced by this machine; the resultant photocopy was light. Additionally, raggedness and breaks in strokes had appeared in subsequent generation of copies.

Sharp AR series. Low speed desktop copiers. Breaks and raggedness were found in subsequent photocopy generations. Pixilation appeared in G1 in diagonal and curved strokes. First and second generation copies could be considered good enough for analysis of handwriting strokes.

Xerox VL 7030. An advanced third generation copier. Noticeable artifacts were absent in all photocopy generations (G1-G5). Fine line quality features such as ink gooping, hesitations, retouching, pen lift were identified in all generations of copies. Overall line quality was found good enough for forensic analysis and comparison in G1 to G5 (Figure 3).
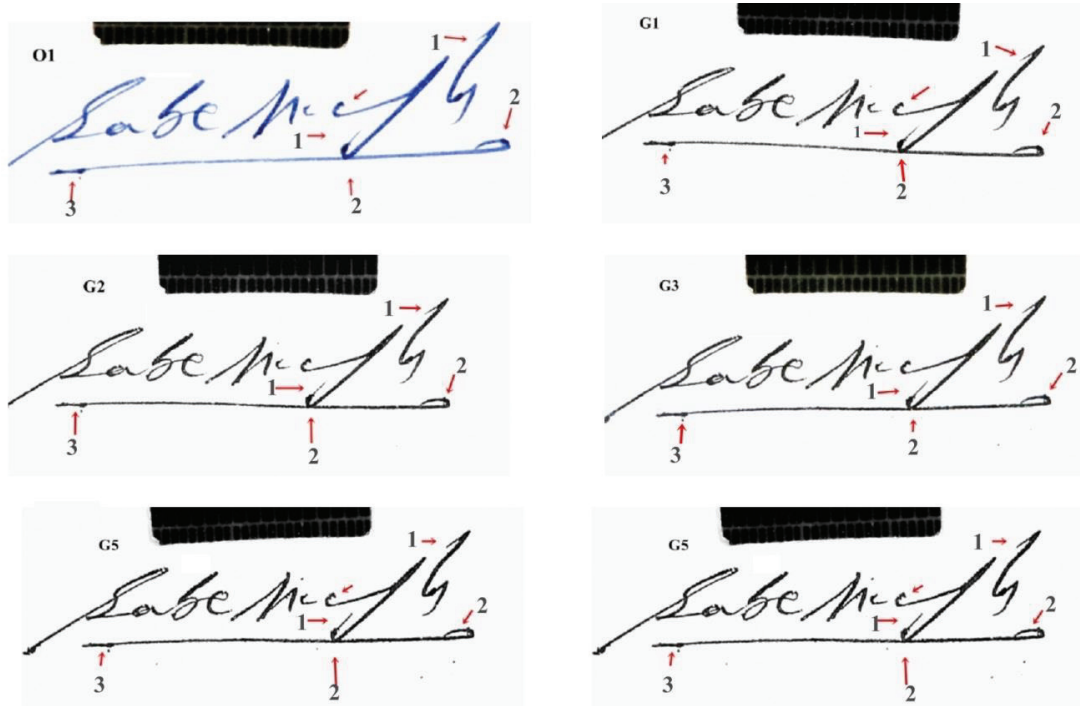

Figure 3. Showing line quality characteristics in the original (O1) and photocopy generations (G1 to G5) of a signature; line features marked as: (1) fine tapered strokes, (2) ink goopings, (3) excessive ink deposition could be identified in G1 to G5 (Xerox VL 7030) 
Xerox DC 440. A medium speed floor standing copier. Extra smoothening and darkening in line strokes was found in photocopies, thus natural irregularities of original line edges were lost in photocopies, specifically in G2 and higher generations. Usually fine line quality features such as ink gooping, hesitation, retouching, excessive ink deposition were lost in or after the second generation of photocopy (G2). However, G1 and G2 photocopies were found suitable for forensic analysis of line quality features handwriting (Figure 4).
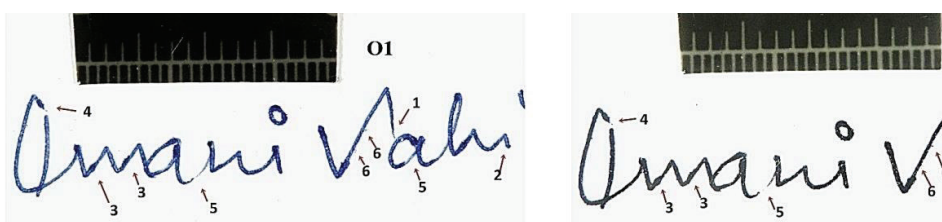

G1
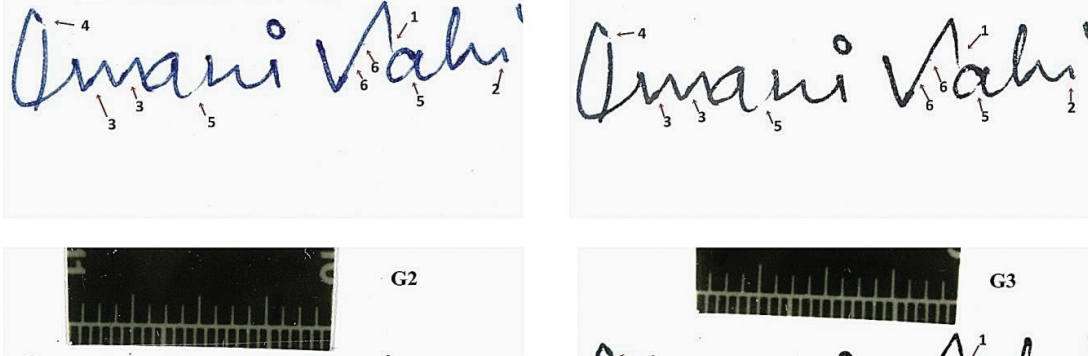

G2

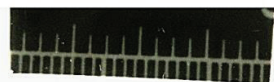

G3
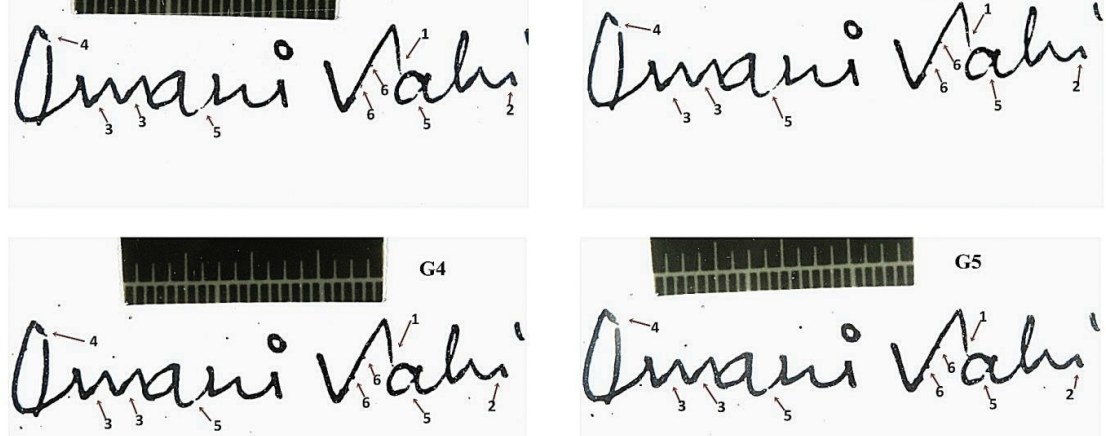

Figure 4. Showing line darkening in photocopy generations (G1 to G5). Line edges are smooth up to G5; line quality features marked as: (1) tapering, (2) blunt ending, (3) and (4) ink goopings, are lost in G3 and higher generations; (5) pen skipping is changed to breaks in G1 to G5, (6) ink traces along the line side are merged and changed to dents

(Xerox DC 440) 
Xerox WC Series. This series included some high speed floor standing copiers (55 to $90 \mathrm{cpm}$ ). The overall line quality was found to be similar to Xerox DC 440.

Canon NP 3050. A desktop analogue copier. It generally produced poor quality photocopies. Breaks in light inked strokes were seen in G1, but overall the line quality was found suitable for forensic analysis in G1.

\section{Discussion}

3.1. Reasons for poor line quality in subsequent photocopy generations produced by portable desktop printers

Printing resolution and the photoconductor drum quality are the main factors causing variation in quality of photocopies produced by small, medium, and high volume copiers. Single layer organic photoconductors (OPCs) used in low speed printers are less expensive and less sensitive than the dual layer OPCs used in high speed printers or copiers. ${ }^{4}$ Photocopiers of several makes could also differ, to some extent, in their print quality due to structural difference in the toner fixing unit. ${ }^{5}$ Medium and high speed floor standing copiers have advanced quality toner fixing unit compared to the low speed portable desktop printers.

3.2. Reasons for good line quality in subsequent photocopy generations produced by advanced generations copiers

Local or universal defects, which could be found in the form of spots and patches resulting from a printer's malfunctions such as foreign contamination on glass platen and photoconductor drum, often degrade the photocopy quality. ${ }^{6}$ However, most of the advanced multifunction copiers have integrated systems/computer programs for detecting and re-

${ }^{4}$ D.S. Weiss, M. Abkowitz, "Advances in organic photoconductor technology", Chemical Reviews 110, 2010, no. 1, pp. 479-526.

5 D. Thompson, D. Tyagi, "Fusing technologies and toner materials relationships", slideshow presented at NIP 24: 24th International Conference on Digital Printing Technologies, Society for Imaging Science \& Technology, 6-11 September 2008, Pittsburgh, PA.

${ }^{6} \mathrm{~J}$. Wang et al., "Local defect detection and print quality assessment", Electronic Imaging 2016, no. 13, pp. 1-7. 
moving some of the local defects. ${ }^{7}$ These advancements in photocopier technology may be one of the reasons for better photocopy quality.

\subsection{Cause of variation in quality of photocopies produced} by same-technology based photocopiers

The input image coming from the reading unit passes through several steps, such as shading correction, enlargement or reduction, edge emphasis, editing, and binary processing. These image processing steps vary depending on the manufacturer and model of a photocopier (Working manual, Canon IR 7200, Xerox WC 5755). Some abrasive actions (cleaning, development, and separation steps) in the copy cycle continuously reduce the thickness of charge transfer layer (CTL). This reduction in thickness of CTL reduced the chargeability of the OPC drum. ${ }^{8}$

\section{Conclusion}

The present study clearly reveals that photocopy quality improves with advancements in the photocopier technology. Line quality features of handwriting could be analysed up to fifth copy generations. The findings of the present study reinforce the necessity of innovation in portable photocopier technology towards continuous improvement of the photocopy quality for forensic analysis of handwriting line quality features.

\section{References}

Allen M., Foundations of forensic document analysis: Theory and practice, Chichester 2016.

Hilton O., Scientific examination of questioned documents, Boca Raton, FL, 1993.

Koppenhaver K.M., Forensic document examination: Principles and practice, Totowa, NJ 2007.

7 R.L. Read, Mapping defects or dirt dynamically affecting an image acquisition device, patent no. US635072A, 2000; I. Pitts, D.I. Stevenson, System for minimizing image defects in a hard-copy input scanner, patent no. US6522431B1, 2003.

8 RICOH CO., LTD, OPCs (Organic Photoconductors), 1995, https://www.hobbielektronika.hu/forum/getfile.php?id=125448 (accessed: 8.04.2020). 
Morris R., Forensic handwriting identification, fundamental concepts and principles, San Diego 2000.

Pitts I., Stevenson D.I., System for minimizing image defects in a hard-copy input scanner, patent no. US6522431B1, 2003.

Read R.L., Mapping defects or dirt dynamically affecting an image acquisition device, patent no. US635072A, 2000.

RICOH CO., LTD, OPCs (Organic Photoconductors), 1995, https://www.hobbielektronika.hu/forum/getfile.php?id=125448 (accessed: 8.04.2020).

Thompson D., Tyagi D., "Fusing technologies and toner materials relationships", slideshow presented at NIP 24: 24th International Conference on Digital Printing Technologies, Society for Imaging Science \& Technology, 6-11 September 2008, Pittsburgh, PA.

Wang J. et al., "Local defect detection and print quality assessment", Electronic Imaging 2016, no. 13.

Weiss D.S., Abkowitz M., "Advances in organic photoconductor technology”, Chemical Reviews 110, 2010, no. 1. 